\title{
Substrate binding accelerates the conformational transitions and substrate dissociation in multidrug efflux transporter AcrB
}

\author{
Beibei Wang ${ }^{1}$, Jingwei Weng ${ }^{1 *}$ and Wenning Wang ${ }^{1,2 *}$ \\ 1 Shanghai Key Laboratory of Molecular Catalysis and Innovative Materials, Department of Chemistry, Fudan University, \\ Shanghai, China, ${ }^{2}$ Institutes of Biomedical Sciences, Fudan University, Shanghai, China
}

\section{OPEN ACCESS}

Edited by:

Attilio Vittorio Vargiu,

Universita' di Cagliari, Italy

Reviewed by:

Ulrich Kleinekathöfer,

Jacobs University Bremen, Germany

Thomas H. Schmidt,

University of Bonn, Germany

*Correspondence: Jingwei Weng and Wenning Wang,

Department of Chemistry,

Fudan University,

220 Handan Road,

Shanghai 200433, China jwweng@fudan.edu.cn; wnwang@fudan.edu.cn

Specialty section:

This article was submitted to

Antimicrobials, Resistance and

Chemotherapy, a section of the journal

Frontiers in Microbiology

Received: 05 January 2015 Accepted: 27 March 2015

Published: 13 April 2015

Citation:

Wang B, Weng J and Wang W (2015)

Substrate binding accelerates the

conformational transitions and

substrate dissociation in multidrug efflux transporter AcrB.

Front. Microbiol. 6:302.

doi: 10.3389/fmicb.2015.00302
The tripartite efflux pump assembly AcrAB-TolC is the major multidrug resistance transporter in E. coli. The inner membrane transporter AcrB is a homotrimer, energized by the proton movement down the transmembrane electrochemical gradient. The asymmetric crystal structures of AcrB with three monomers in distinct conformational states [access (A), binding (B) and extrusion (E)] support a functional rotating mechanism, in which each monomer of AcrB cycles among the three states in a concerted way. However, the relationship between the conformational changes during functional rotation and drug translocation has not been totally understood. Here, we explored the conformational changes of the AcrB homotrimer during the ABE to BEA transition in different substrate-binding states using targeted MD simulations. It was found that the dissociation of substrate from the distal binding pocket of $\mathrm{B}$ monomer is closely related to the concerted conformational changes in the translocation pathway, especially the side chain reorientation of Phe628 and Tyr327. A second substrate binding at the proximal binding pocket of A monomer evidently accelerates the conformational transitions as well as substrate dissociation in B monomer. The acceleration effect of the multi-substrate binding mode provides a molecular explanation for the positive cooperativity observed in the kinetic studies of substrate efflux and deepens our understanding of the functional rotating mechanism of AcrB.

Keywords: AcrB, MD simulation, substrate binding, allosteric effect, drug extrusion, positive cooperativity

\section{Introduction}

In Gram-negative bacteria, resistance-nodulation-division (RND) superfamily proteins play a major role in the efflux of a wide range of antibiotics and toxic compounds out of cell. RND superfamily, together with other classes of multidrug efflux pumps, constitute one of the major mechanisms of multidrug resistance (MDR) in bacteria, which represents a serious impediment to improved healthcare (Higgins, 2007). RND transporter is embedded in the inner membrane and works as a drug-proton antiporter. Fueled by proton diffusion down the transmembrane (TM) electrochemical gradient, it collects substrates from the periplasm or the inner leaflet of inner membrane and extrudes them to the lumen of outer membrane component (Nikaido, 1996). The RND transporter AcrB in Escherichia coli has been extensively studied as a prototype of the family, which 
exports a number of dyes, detergents, chloramphenicol, tetracyclines, macrolides, $\beta$-lactams, fluoroquinolones, and organic solvents. It functions in the form of AcrAB-TolC tripartite complex (Zgurskaya and Nikaido, 1999b; Tikhonova and Zgurskaya, 2004; Collu et al., 2012; Du et al., 2014) by working collaboratively with the periplasmic adaptor protein AcrA (Zgurskaya and Nikaido, 1999a; Mikolosko et al., 2006) and the outer membrane protein TolC (Koronakis et al., 2000). As the core of the complex, AcrB is responsible for substrate recognition and energy supplement (Elkins and Nikaido, 2002).

Crystallographic studies have revealed the structure of AcrB as a homotrimer (Murakami et al., 2002, 2006; Seeger et al., 2006). Each monomer is composed of a TM domain, a porter domain and a TolC-docking domain (Figure 1A). The TM domain contains $12 \mathrm{TM}$ helices and encompasses a putative proton relay pathway lined by Asp407 and Asp408 on TM4 helix, Lys940 on TM10 helix and Thr978 and Arg971 on TM11 helix, which harvests proton motive force from the transmembrane electrochemical gradient (Murakami et al., 2006; Seeger et al., 2006, 2009; Su et al., 2006; Takatsuka and Nikaido, 2006). The porter domain and the TolC docking domain are folded by two periplasmic loops of the TM domain, one between TM1 and TM2 helices and the other between TM7 and TM8 helices. The porter domain could be further divided into four subdomains PN1, PN2, PC1, and PC2 (Figure 1B), the inter-domain space between which forms the pathway for substrate translocation (Sennhauser et al., 2007; Husain and Nikaido, 2010; Nakashima et al., 2011; Yao et al., 2013). Despite of the diversity of entrances (Husain and Nikaido, 2010; Nakashima et al., 2011; Yao et al., 2013), substrates are found to constantly pass through the cleft between PC1 and PC2, the "switch-loop" (also called Phe-617 loop, Gloop), the cavity enclosed by PC1, PN1, and PN2, and the exit constricted by Gln124 (on PN1 subdomain) and Tyr758 (on the TolC-docking domain) (Figure 1B) during the translocation process, and finally enter the central funnel enclosed by the TolC-docking domains. Two binding pockets have been identified along the translocation pathway inside the porter domain. The pocket more distal from the entrances, i.e., the distal binding pocket (DBP), lies between PC1 and PN2 and is rich in aromatic residues including Phe610, Phe615, Phe617, and Phe628 on PC1 and Phe136 and Phe178 on PN2 (Figure 1B) (Murakami et al., 2006; Nakashima et al., 2011; Eicher et al., 2012). The proximal binding pocket (PBP, also called access pocket) between PC1 and PC2 is more hydrophilic (Figure 1B), capturing substrates through a combination of hydrogen bond, hydrophobic and $\pi$ $\pi$ stacking interactions (Nakashima et al., 2011; Eicher et al., 2012).

AcrB is highly dynamic and its conformational changes are essential for its transport activity (Takatsuka and Nikaido, 2007, 2009; Seeger et al., 2008). Three major conformational states of monomers have been identified in the asymmetric crystal structures (Murakami et al., 2006; Seeger et al., 2006; Sennhauser et al., 2007; Nakashima et al., 2011; Eicher et al., 2012). The access (A, also called loose, L) and binding (B, also called tight, $\mathrm{T}$ ) states both have an opened PC1/PC2 cleft and an occluded exit, but differs in the binding pockets. The DBP in B state is intact and capable of accommodating substrates, whereas in A state, the DBP partially collapses and the PBP is found capable of substrate binding instead (Nakashima et al., 2011; Eicher et al., 2012). The extrusion (E, also called open, O) state adopts a closed PC1/PC2 cleft and an opened exit for substrate extrusion. Edified by the asymmetric crystal structures, each monomer of AcrB trimer is believed to undergo a succession of transitions among the three states during transportation, cycling from $\mathrm{A}$ to $\mathrm{B}$ to $\mathrm{E}$ and back to $\mathrm{A}$ state, i.e., the functional rotating mechanism (Murakami et al., 2006; Seeger et al., 2006; Pos, 2009). The mechanism also predicts that the transition of each monomer is dependent on the conformations of its neighbors. When A monomer (originally staying in A state) evolves to B state, $\mathrm{B}$ and $\mathrm{E}$ monomers would respectively switch into $\mathrm{E}$ and $\mathrm{A}$ states simultaneously, accomplishing one $\mathrm{ABE} \rightarrow \mathrm{BEA}$ transition step.

The functional rotating mechanism implicates highly cooperative conformational changes among the three monomers. Subsequent kinetic studies indeed revealed positive cooperativity in substrate efflux (Nagano and Nikaido, 2009; Lim and Nikaido, 2010). However, the underlying detailed picture of the functional rotating and the cooperativity in substrate efflux remain elusive, especially the relationship between the conformational changes in AcrB and the kinetic behavior of substrate efflux. Molecular dynamics (MD) simulation is a powerful tool in providing high spatiotemporal resolution details for conformational changes of proteins. MD simulations have been used to reveal the dynamics of RND transporters (Fischer and Kandt, 2013; Yamane et al., 2013), the movement of substrate (Fischer and Kandt, 2011; Feng et al., 2012) and the interaction network between the transporter and substrate (Vargiu and Nikaido, 2012; Kinana et al., 2013). Due to the sampling efficiency problem of the conventional MD simulation, some enhanced sampling protocols, such as targeted MD (Ma and Karplus, 1997; Kong et al., 2002; van der Vaart et al., 2004; Compoint et al., 2005; Cheng et al., 2006; Weng et al., 2010, 2012), were also implemented to extract details of the conformational transition in one functional rotating step (Schulz et al., 2010, 2011; Vargiu et al., 2011). In this work, we performed targeted MD simulations to investigate the conformational transition with different number of binding substrates. Binding of one or two molecules of doxorubicin show similar concerted conformational changes in the translocation pathway and the dissociation of substrate from the DBP. A close correlation was observed between side chain reorientations of Phe628 and Tyr327 and substrate dissociation in both systems. Binding of a second substrate evidently facilitates the conformational changes in the translocation pathway as well as the dissociation of the DBP-bound substrate, explaining the positive cooperativity in substrate transportation.

\section{Materials and Methods}

\section{System Setup}

The crystal structure of AcrB (PDBID: 2GIF) was used as the starting structure of all simulations. In the crystal structure, residues 1034-1049 are missing in chain A and C, and residues 1046-1049 are missing in chain B. To keep the 3-fold symmetry of the protein, we truncated the residues 1034-1045 in chain 


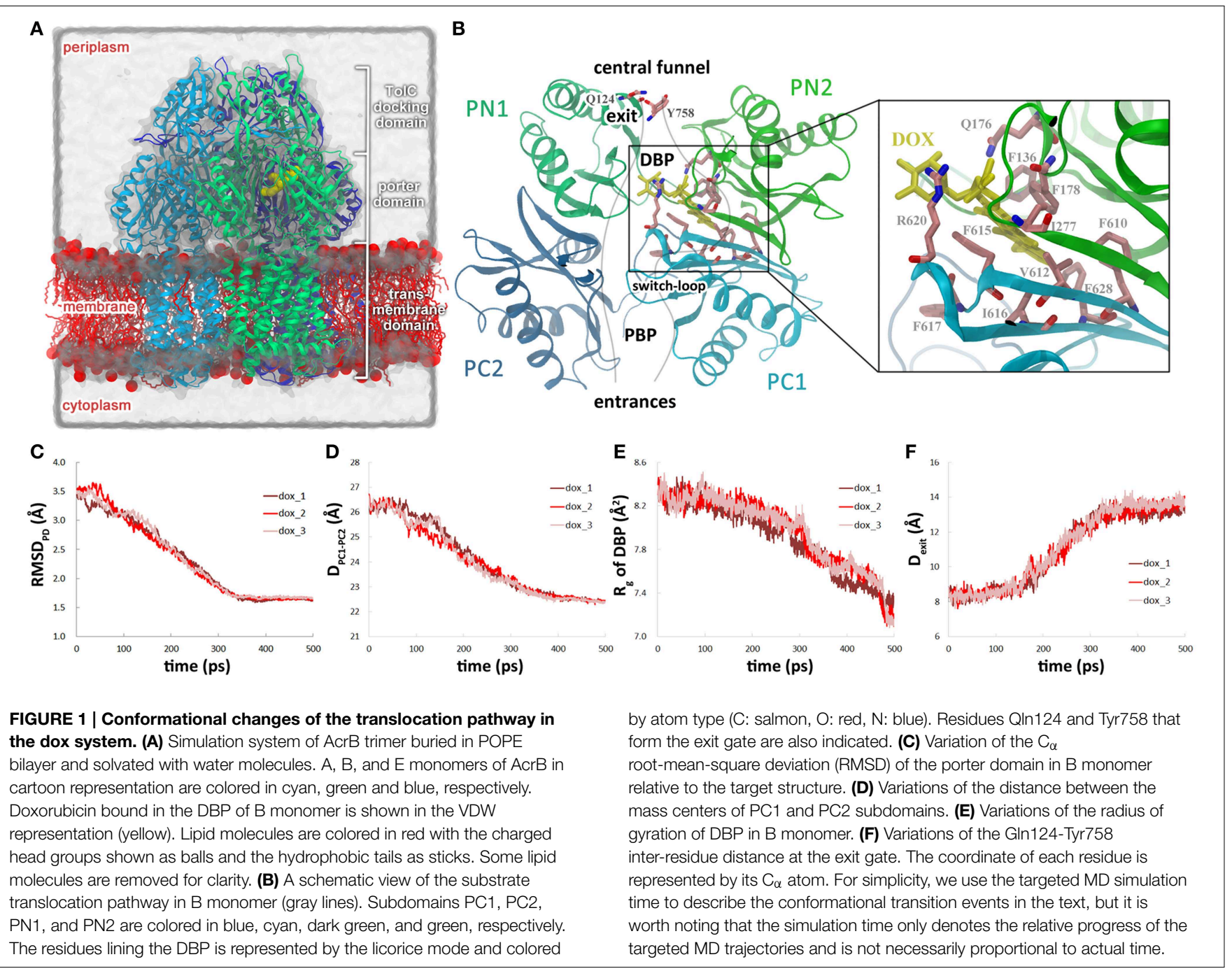

B. Since no substrate was cocrystallized in this structure, one doxorubicin was docked into the DBP of B monomer according to the AcrB-doxorubicin complex structure (PDBID: 2DR6). In the simulations with two substrates, the second doxorubicin was docked into the PBP of A monomer by using Autodock (Morris et al., 2009). Default parameters were used for the docking procedure and the center structure of the largest cluster was selected for the simulations.

The AcrB-doxorubicin complex was then buried into a preequilibrated palmitoyloleoylphosphatidylethanolamine (POPE) bilayer consisting of 512 lipid molecules following the "shrinking" method (Kandt et al., 2007). Additional 10 lipid molecules (5 lipids in the outer leaflet and 5 in the inner leaflet) were manually placed in the central cavity enclosed by the three TM domains. The number of lipids was estimated by the area of the cavity calculated by Hole (Smart et al., 1996) divided by the area per lipid for POPE (59 $\AA^{2}$ ) (Rappolt et al., 2003). The protein-lipids complex were solvated with a rectangular box of water and neutralized by $42 \mathrm{Na}^{+}$ions. The box size is $140 \times 140 \times 160 \AA^{3}$ to keep any atom of protein at least $10 \AA$ away from the edge of the box. The simulation system contains 320,557 atoms in total (Figure 1A).

\section{Simulations}

All simulations were carried out with the parallel MD package NAMD 2.7 (Phillips et al., 2005) with CHARMM27 force field (Feller et al., 1997; MacKerell et al., 1998, 2004). TIP3P model (Jorgensen et al., 1983) was used for water molecules. The CGenFF force field parameters of doxorubicin were derived by using the ParamChem tool (Vanommeslaeghe et al., 2010, 2012; Vanommeslaeghe and MacKerell, 2012). All titratable residues were kept in their default protonation states except Asp407 and Asp408. For these two residues, two different protonation schemes were used. In the first scheme, the protonation states were set according to the asymmetric structure of AcrB (Murakami et al., 2006), i.e., Asp407 and Asp408 were deprotonated in the $\mathrm{A}$ and $\mathrm{B}$ monomers, and protonated in the $\mathrm{E}$ monomer. In the second scheme, Asp407 and Asp408 were protonated in the $\mathrm{B}$ monomer and deprotonated in the $\mathrm{A}$ and $\mathrm{E}$ monomers. The system was maintained at 1.01325 bar by using 
the Nosè-Hoover Langevin piston method (Martyna et al., 1994; Feller et al., 1995) and at $300 \mathrm{~K}$ by Langevin dynamics with a damping coefficient of $1.0 \mathrm{ps}^{-1}$. Periodic boundary conditions were employed, and the electrostatic interactions were evaluated using the particle-mesh Ewald (PME) method (Darden et al., 1993 ) with a cutoff of $12 \AA$ and a grid spacing of $1 \AA$. The van der Waals interactions were switched at $10 \AA$ and truncated at 12 Å. All bonds involving hydrogen atoms were constrained by the SETTLE algorithm (Miyamoto and Kollman, 1992). A time step of $2 \mathrm{fs}$ was used for the conventional MD simulations, and $1 \mathrm{fs}$ was used for the targeted MD simulations.

The whole system was equilibrated for $2 \mathrm{~ns}$ with the protein restrained by harmonic forces (the force constant was set to 1000 $\mathrm{kcal} / \mathrm{mol} / \AA^{2}$ ), followed by a 4 ns run without any restraint. AcrB was stable in the $4 \mathrm{~ns}$ NPT run with its $\mathrm{C}_{\alpha}$ root-mean-square deviation (RMSD) relative to the initial crystal structure fluctuating around $2.2 \AA$ in the last 2 ns. The final structure of the unbiased MD run was used as the initial structure of the targeted MD simulations. The target structure was obtained by setting each monomer to the state of the next functional rotating step, i.e., $\mathrm{ABE} \rightarrow \mathrm{BEA}$.

Targeted MD method propels a known initial structure to a known target structure by using an external potential (Schlitter et al., 1993). The potential decreases the RMSD of the system relative to the target structure toward a preset value at each time step. The potential can be described as:

$$
U_{T M D}=\frac{1}{2} \frac{k}{N}[R M S D(t)-R M S D *(t)]^{2}
$$

where $R M S D(t)$ is the instantaneous best-fit RMSD of the current coordinates to the target coordinates, $R M S D^{*}(t)$ is the preset RMSD value for the current time step, $k$ is the force constant and $N$ is the number of targeted atoms. The external forces were casted on all heavy atoms of AcrB. A tclForce script was used to perform mass-weighted targeted MD simulations. To assess the influence of parameters on the results, we performed a series of simulations using different force constants $(k / N=1,2$, and 3 $\mathrm{kcal} / \mathrm{mol} / \AA^{2}$ ) and different simulation times (500 ps and $1 \mathrm{~ns}$ ) (Table S1, Supplemental Data).

\section{Data Analyses}

The radius of gyration $\left(R_{g}\right)$ of the DBP is defined by all the atoms in the residues $628,610,136,178,615,617,277,626,620,176$, and 612 which lies in the vicinity of DBP:

$$
R_{g}=\left(\sum_{i=1}^{n} \omega(i)\left(r(i)-r_{m c}\right)^{2}\right) /\left(\sum_{i=1}^{n} \omega(i)\right)
$$

where $r(i)$ is the position of the $i$ th atom, $\omega(i)$ is the mass weight and $r_{m c}$ is the weighted center of the selected atoms. The distance between Gln124 \& Tyr758 is defined as the distance between the $\mathrm{C}_{\alpha}$ atoms of the two residues. All data analysis and molecular structure visualization were conducted by VMD (Humphrey et al., 1996).

\section{Results}

We employed targeted MD simulations to acquire an atomistic view of the conformation changes of AcrB in one functional rotating step. The conformational changes were studied with one doxorubicin bound in the DBP of B monomer (denoted as dox hereafter), or with two doxorubicins bound in the DBP of $\mathrm{B}$ monomer and in the PBP of A monomer, respectively (denoted as 2dox hereafter). Three parallel trajectories started with different initial velocities were produced for each system. The results were found to be insensitive to the selection of force constants or time lengths (Table S1, Supplemental Data, see below for details). In the following, we will first introduce the collective and local conformational changes in the translocation pathway and the association of these changes with doxorubicin movement. Then we focus on the effect of the presence of the second doxorubicin by comparing the trajectories of the dox and 2dox systems. For simplicity, we use the targeted MD simulation time to describe the conformational transition events in the text, but it is worth noting that the simulation time only denotes the relative progress of the targeted MD trajectories and is not necessarily proportional to actual time.

\section{Concerted Conformational Changes in the Translocation Pathway of B Monomer}

At the beginning of the simulation, large-scale conformational changes were observed in the porter domain of the $\mathrm{B}$ monomer (Figure 1B). In all three trajectories of the dox system, the $\mathrm{C}_{\alpha}$ RMSDs of the porter domain decreased linearly relative to the targeted structure in the first 330 ps, after which the RMSD profiles fluctuated around $1.7 \AA$ till the end of the simulations (Figure 1C). The variations of the porter domain are largely resulted from the relative motions between the subdomains. The motions reduced the distance between the mass centers of PC1 and PC2 subdomains from 26.6 to $22.8 \AA$ in 330 ps (Figure 1D), closing the PC1-PC2 cleft and occluding the translocation pathway toward the entrance (Figure 1B). On the other hand, the DBP shrunk from $\sim 100$ ps, with the $R_{g}$ decreasing till the end of the simulations (Figure 1E). The decline of $R_{g}$ indicates contraction of the middle part of the translocation pathway which would contribute to the dissociation of substrate (see below). During this period, the distance between residues Gln124 and Tyr758, which constrict the exit of drug extrusion (Yao et al., 2013) (Figure 1B) increased from 8.7 to $13.1 \AA$ in about 180 ps (Figure 1F), resulting in the opening of the exit. It is worth noting that the timing of these conformational changes obviously overlap with each other, with the exit gate opening at 155-340 ps (Figure 1F), the PC1/PC2 cleft closing at 50-330 ps (Figure 1D) and the DBP shrinking at 75-500 ps (Figure 1E), indicating concerted conformational motions of the translocation pathway in the first 330 ps of the simulations.

\section{Dissociation of Doxorubicin from the DBP}

As the concerted transmutation of the translocation pathway started in the first 155 ps, the substrate kept stably bound inside the DBP through hydrophobic and $\pi-\pi$ interactions with the protein (Figure S1, Supplemental Data). The binding mode in 


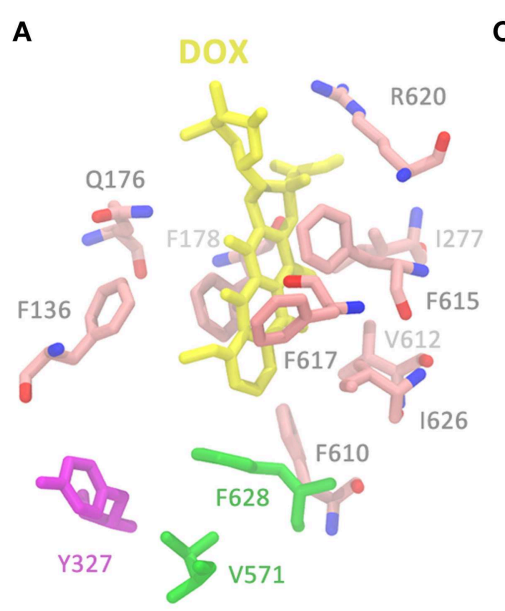

initial structure

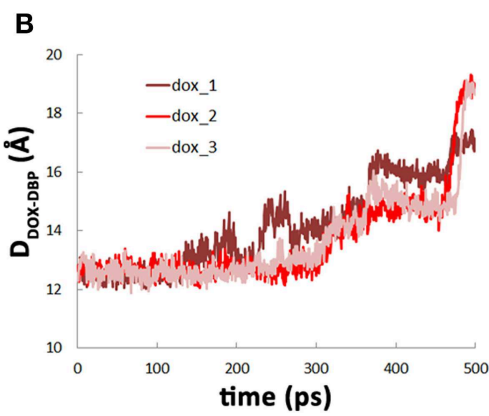

C

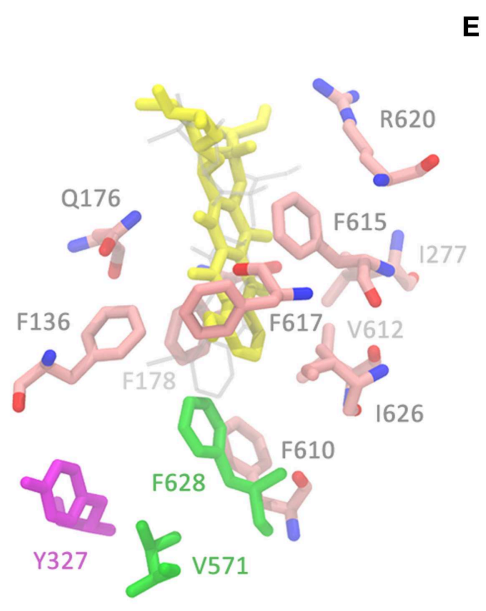

$230 \mathrm{ps}$

D

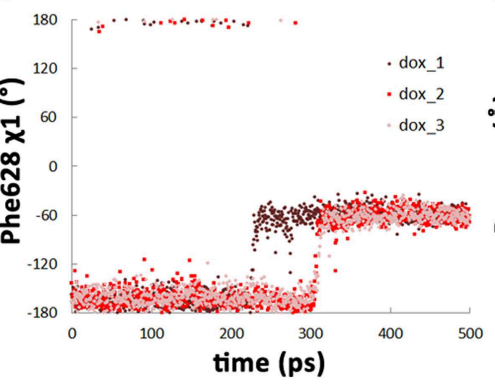

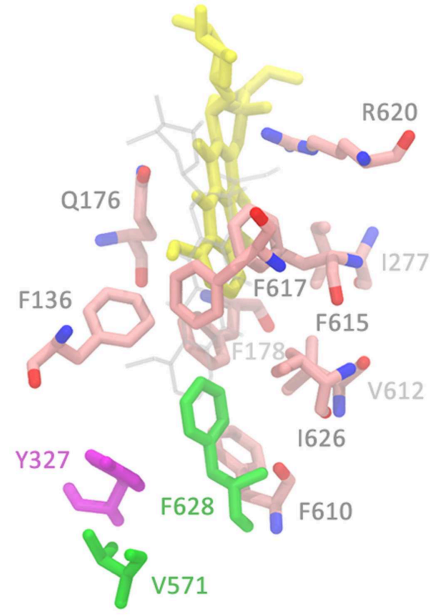

end structure

$\mathbf{F}$

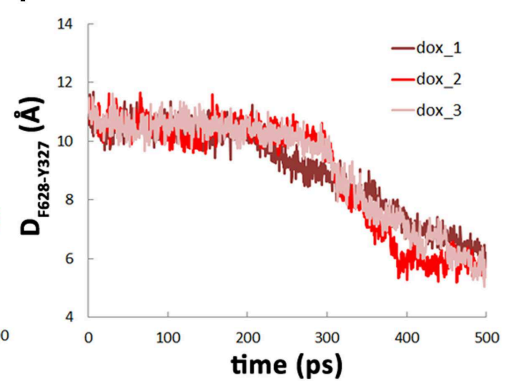

FIGURE 2 | Dissociation of doxorubicin from the DBP in the dox system. (A) Close view of doxorubicin (yellow) bound in DBP in the initial structure. Residues lining the DBP are colored by atom type (C: salmon, O: red, N: blue), except Phe628, Val571, and Tyr327, which are highlighted by green and purple. (B) Variations of the distance between doxorubicin and the DBP with simulation time. (C) Snapshot of doxorubicin in DBP at 230 ps in one of the trajectories when Phe628 reoriented and pointed toward the substrate. (D) Variations of $\chi 1$ angle of Phe628 along the simulation trajectories. (E) Close view of doxorubicin and the residues lining DBP at the end of the simulation when Tyr327 inserted in between Phe628 and Val571. (F) Variations of the distance between the mass center of Tyr327 side chain and the $\mathrm{C}_{\alpha}$ atom of Phe628. the simulations is slightly different from the two known binding modes presented in crystal structures (Murakami et al., 2006; Eicher et al., 2012). The position of the amino-sugar moiety was almost invariant, whereas the aglycone moiety showed a new posture by extending directly toward Phe628 rather than pointing toward Phe610 (Eicher et al., 2012) or Phe617 (Murakami et al., 2006) in the crystal structures (Figure S1, Supplemental Data). The diversity of binding mode could be attributed to the differences in the local configuration of the DBP in different crystal structures (see Methods). Similar structural dependence of binding mode was also reported for chloramphenicol in the DBP of AcrB (Vargiu and Nikaido, 2012).

The stable interactions between doxorubicin and DBP were disrupted at 225-300 ps as doxorubicin detached from the binding site. The dissociation of doxorubicin was monitored by the distance between the mass center of doxorubicin and the $C_{\alpha}$ atom of Phe628 which lies at the bottom of the DBP (Figure 2A). Two of the trajectories exhibit very similar profiles of substrate motions. The doxorubicin initially stayed in the DBP with the distance fluctuating around $12.5 \AA$, and detached from the binding site after 300 ps as the distance increased (Table1, Figure 2B, pink and red lines). In another trajectory, more evident fluctuations were observed before dissociation, such as the peak at $225 \mathrm{ps}$ on the profile (Figure 2B, brown line). After 275 ps, doxorubicin became fully dissociated from the DBP. At the end of the simulations, a displacement of 5-7 $\AA$ toward the exit could be observed and the dissociation is irreversible, as verified by the extended 2-ns simulations with all restraining forces removed (Figure S2, Supplemental Data). Similar extrusion process was also observed by Vargiu et al. using targeted MD simulations (Vargiu et al., 2011).

\section{Correlation between the Conformational Changes in the DBP and Doxorubicin Dissociation}

Further inspection of the trajectories show that the dissociation process of doxorubicin is closely related to the conformational changes of the DBP, especially the side chain orientation of Phe628. The benzyl group of Phe628 originally pointed toward the TM domain at the beginning of the simulations, characterized by the $\chi 1$ angle of about $-160^{\circ}$ (Figure 2A), and then it switched to a new orientation with the $\chi 1$ angle at 
TABLE 1 | Conformational changes associated with the dissociation of doxorubicin.

\begin{tabular}{lcccc}
\hline System $^{\mathbf{a}}$ & Trajectory & $\begin{array}{c}\text { Time of } \\
\text { doxorubicin } \\
\text { dissociation } \\
\mathbf{( p s}^{\mathbf{b}}\end{array}$ & $\begin{array}{c}\text { Time of Phe628 } \\
\text { side chain } \\
\text { reorientation } \\
\mathbf{( p s}^{\mathbf{c}}\end{array}$ & $\begin{array}{c}\text { Time of Tyr327 } \\
\text { side chain } \\
\text { insertion (ps) }\end{array}$ \\
\hline dox & 1 & 225,275 & 225 & $235-500$ \\
& 2 & 300 & 300 & $300-395$ \\
& 3 & 300 & 300 & $300-470$ \\
\hline $\mathbf{2 d o x}$ & 1 & 200 & 200 & $200-400$ \\
& 2 & 225 & 225 & $225-370$ \\
& 3 & 190 & 190 & $190-420$ \\
\hline
\end{tabular}

${ }^{a}$ dox denotes the simulation system with only one doxorubicin bound in $D B P$ of $B$ monomer, 2dox denotes the simulation system with a second doxorubicin bound in PBP of $A$ monomer.

${ }^{b}$ Time when doxorubicin detached from the binding pocket. Temporary dissociation leads to more than one dissociation time.

cTime when Phe628 reoriented its side chain and pointed toward the substrate.

dTime period of the decreasing of the distance between Tyr327 side chain and Phe628 $C_{\alpha}$.

about $-60^{\circ}$ (Figures 2A,C,D). The reorientation of Phe628 side chain occurred at 300 ps in two of the trajectories (Figure 2D, pink and red lines) and at 225 ps in the other (Figure 2D, brown line), well consistent with the departure time of doxorubicin (Table 1). The close correlation between the two events can be rationalized by the steric hindrance between the bulky side chain of Phe628 and the doxorubicin at binding site (Figure 2C), which pushes the substrate from the binding pocket. The importance of Phe628 for substrate dissociation was previously reported by biochemical studies, in which substitution of the bulky side chain reduced the resistance to doxorubicin and other substrates (Husain and Nikaido, 2010; Nakashima et al., 2011).

After the reorientation of Phe628, local conformational rearrangement was observed. The space originally occupied by the benzyl group of Phe628 and the isopropyl group of Val571 (Figures 2A,C) was subsequently filled by the phenol group of Tyr327, which is several residues preceding TM2 helix of the TM domain (Figure 2E). Interestingly, the rearrangement started almost simultaneously with the reorientation of the Phe628 side chain (Figure 2F, Table 1). This implies a functional role of Tyr327 in stabilizing the reorientated conformation of Phe628.

To examine possible influence of TM domain protonation state on substrate dissociation, we produced targeted MD trajectories for AcrB with different protonation state (Table S1, Supplemental Data). It turns out that these trajectories demonstrated very similar features such as substrate dissociation, side chain reorientation of Phe628 and Tyr327 (Figure S3, Table S1, Supplemental Data), indicating that the current simulation protocol is insensitive to the changes in protonation state. Different simulation time range (500 ps or $1 \mathrm{~ns}$ ) and different force constant $\left(k / N=1,2\right.$, or, $\left.3 \mathrm{kcal} / \mathrm{mol} / \AA^{2}\right)$ were also tested. These factors do not disturb the correlation between conformational changes in the DBP and substrate dissociation, either (Table S1, Supplemental Data).

\section{Binding of the Second Doxorubicin Accelerates the Conformational Changes in the Translocation Pathway}

To study the effect of multi-substrate binding on the conformational transition, a second doxorubicin was docked into the PBP of A monomer (see Methods) to build the 2dox system (Figure 3A). The position of the docked substrate closely resembles the binding mode of PC1-proximal doxorubicin (Figure S4, Supplemental Data) observed in the AcrB-doxorubicin complex structure (Eicher et al., 2012). The presence of the second doxorubicin affects the conformational changes in the translocation pathway in several aspects. First, the closing motion of the PC1/PC2 cleft was antedated, illustrated by the $\sim 70$ ps earlier decreasing period of the inter-domain distance in the $\mathbf{2 d o x}$ system than that in the dox system (Figure 3B). Secondly, the conformational changes in the DBP were moved ahead as the $R_{g}$ decreased to $7.8 \AA$ at about 200 ps in the 2 dox system, instead of at 300 ps in the dox system (Figure 3C). Finally, the opening motion of the exit was accelerated as the distance between Gln124 and Tyr758 exceeded $13 \AA$ at 220 ps, rather than at 300 ps in the dox system (Figure 3D). It is worth noting that the dox and 2dox systems share very similar initial and final structures at the PC1/PC2 cleft, the DBP and the exit gate (Figures 3B-D), so that the external forces casted on these regions are generally equivalent in both systems (see Methods). The systematic antedate of these events indicates that the conformational changes in the translocation pathway are evidently accelerated by the binding of the second doxorubicin at A monomer.

\section{Binding of the Second Doxorubicin Facilitates the Dissociation of Doxorubicin}

Along with the antedated conformational changes in the translocation pathway, substrate dissociation in the 2dox system was also earlier than that in the dox system. In the three trajectories, irreversible dissociation occurred at 200, 230, and $190 \mathrm{ps,}$ respectively (Figure 4A, Figure S5, Supplemental Data), about 70 ps earlier than that of the dox system on average. Along with substrate dissociation, the change of Phe628 $\times 1$ angle from -160 to $-60^{\circ}$ and the insertion of Tyr327 in between Phe628 and Val571 were also moved ahead to about 200 ps (Figures 4B,C). Similar with the dox system, there is also a close correlation between substrate dissociation and side chain reorientation of Phe628 and Tyr327 (Table 1) and the trend is kept with varied simulation time range or force constant (Table S1, Supplemental Data), indicating that the 2dox system shares the same mechanism of doxorubicin dissociation in B monomer with the dox system.

\section{Conformational Changes in AcrB Does Not Promote the Transportation of the Second Doxorubicin}

The movement of doxorubicin in the PBP of A monomer during the functional rotation was also examined (Figure 3A). In contrast to the displacement toward the exit observed for the DBP-bound doxorubicin in B monomer, the PBP-bound doxorubicin in A monomer moved in an opposite direction, further away from the DBP by about $2 \AA$ at the end of the simulations (Figure 5A). The slight retrogression of the substrate could be 

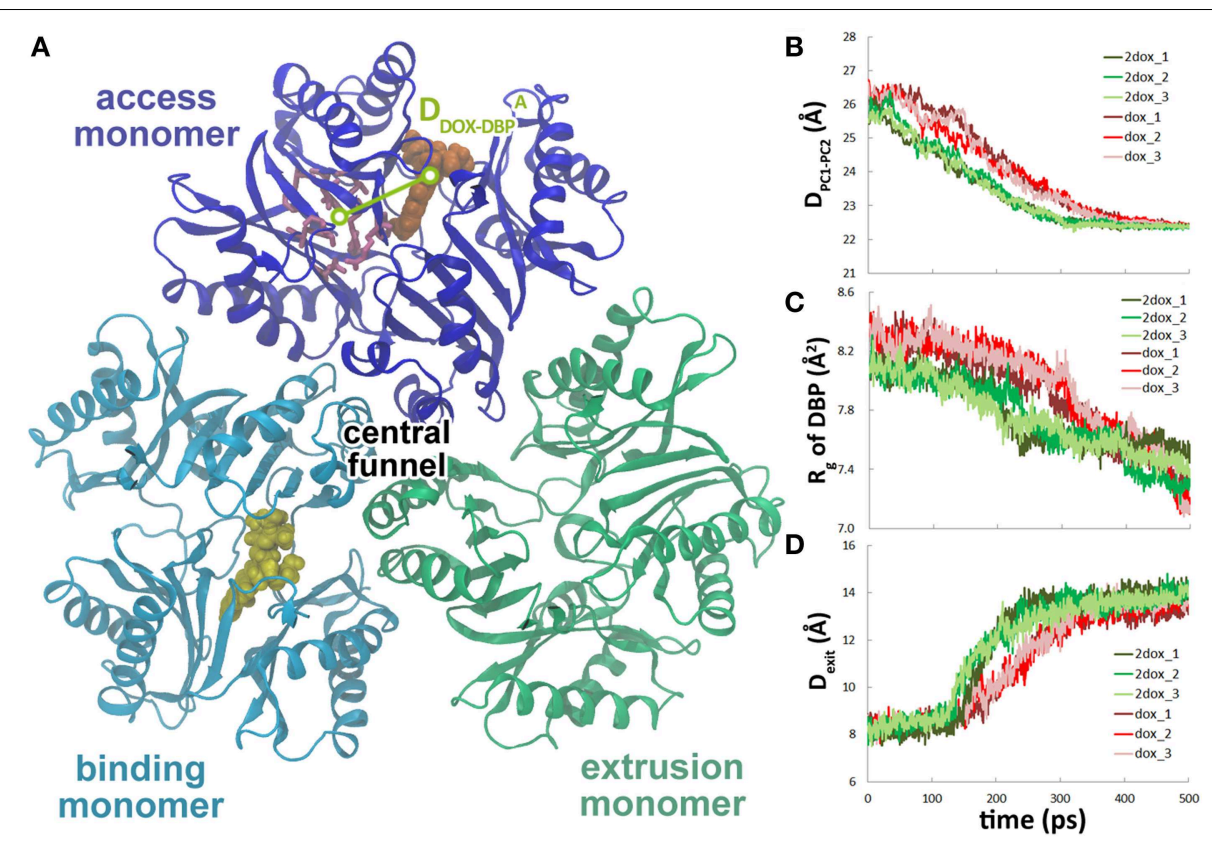

FIGURE 3 | The second substrate binding at A monomer accelerates the conformational changes of $\mathbf{B}$ monomer. (A) Top view of the porter domains with two doxorubicins (yellow and orange) bound in the initial structure of the $\mathbf{2 d o x}$ system. The distance between doxorubicin and DBP in A monomer is indicated as $D_{D O X}^{A}-D B P \cdot(B)$ Comparison of the variations of the distance between the mass centers of PC1 and PC2 subdomains of $\mathrm{B}$ monomer in dox and 2dox systems. (C) Comparison of the variations of $\mathrm{R}_{g}$ of the DBP of B monomer in dox and $\mathbf{2 d o x}$ systems. (D) Comparison of the variations of the Gln124-Tyr758 inter-residue distance at the exit region of B monomer in dox and $\mathbf{2 d o x}$ systems.
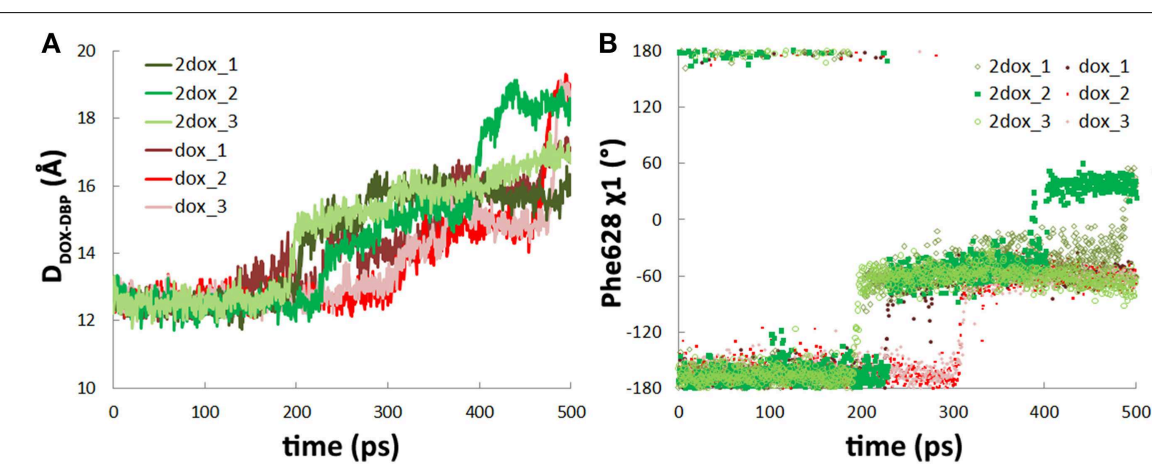

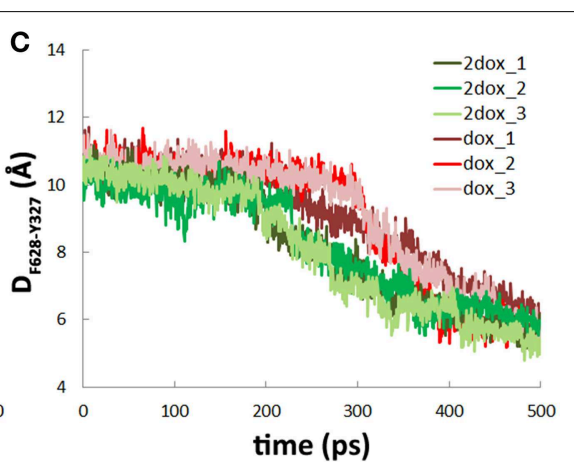

FIGURE 4 | The second substrate binding at monomer A accelerates the dissociation of doxorubicin from the DBP in monomer B. (A) Comparison of the variations of the distance between doxorubicin and the DBP of monomer B in dox and 2dox systems. (B)
Comparison of the variations of $\chi^{1}$ angle of Phe628 in dox and $\mathbf{2 d o x}$ systems. (C) Comparison of the variations of the distance between the mass center of Tyr327 side chain and the $\mathrm{C}_{\alpha}$ atom of Phe628 in dox and 2dox systems. attributed to the inter-domain motion between PC1 and PC2 subdomains. The PC1/PC2 cleft opened by about $1.5 \AA$ when A monomer evolved toward the $\mathrm{B}$ state following the functional rotating mechanism (Figure 5B). The opening motion may weaken the interactions between AcrB and doxorubicin, and leads to the movement of substrate toward the entrance.

\section{Discussion}

The functional rotating mechanism based on the asymmetric structure of AcrB assumes that each monomer cycles among the three conformational states (A, B, and E) concertedly, facilitating the unidirectional translocation of the substrate toward the outer membrane protein TolC. In this work, we simulated one functional rotating step of $\mathrm{AcrB}(\mathrm{ABE} \rightarrow \mathrm{BEA})$ in the context of one or two bound substrates using targeted MD simulations, and provided an atomic spatiotemporal view of the conformational changes and substrate movement.

The targeted MD trajectories demonstrated that the simulation started with conformational changes in the translocation pathway, involving a set of concerted motions such as the closing of the PC1/PC2 cleft (Figure 1D), the shrinking of the DBP 

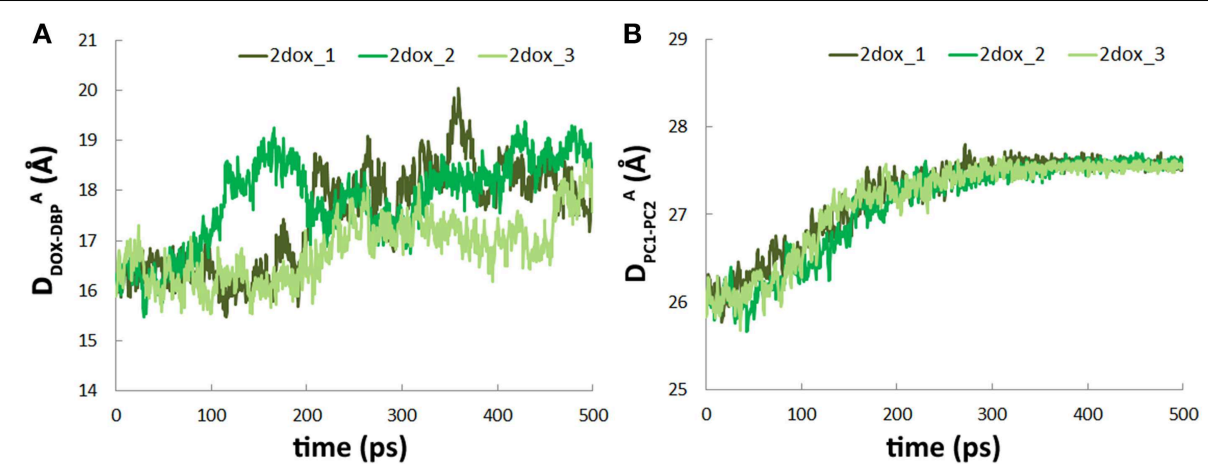

FIGURE 5 | Conformational transition of $\mathbf{A} \rightarrow \mathbf{B}$ does not facilitate substrate translocation from PBP to DBP. (A) Variations of $D_{D O X}^{A}$ DBP along the simulation trajectories. (B) Variations of the distance between the mass centers of PC1 and PC2 subdomains in A monomer.

(Figure 1E), and the opening of the exit gate (Figure 1F). In the following $200 \mathrm{ps}$, however, doxorubicin in DBP did not show evident motions until the dissociation from DBP occurred at 225-300 ps. Therefore, the conformational changes in the translocation pathway are the prerequisite and the driving force for substrate dissociation. Specifically, it has been identified that the side chain reorientation of Phe628 is mainly responsible for substrate dissociation (Figure 2). The importance of Phe628 for substrate transportation is supported by previous biochemical experiments, in which removal of the bulky side chain of Phe628 evidently reduced the resistance to doxorubicin and other substrates of AcrB (Husain and Nikaido, 2010; Nakashima et al., 2011). Similar protein-driven substrate movement was also observed by A. Vargiu et al. using the same method (Schulz et al., 2010; Vargiu et al., 2011) though the atomistic details of proteinsubstrate interaction are slightly different. Due to the limited simulation time in this work, we did not observe the entire extrusion process of the substrate. Hundreds of nanoseconds or more may be needed for the substrate to overcome the barriers on its way to the central funnel (Ruggerone et al., 2013; Yao et al., 2013).

The functional rotating mechanism of AcrB assumes that the conformational transitions of the three monomers occur in a concerted way, implicating cooperativity in substrate translocation. Kinetic studies of substrate transport of AcrB in intact cells identified positive cooperativities in the efflux of various cephalosporins (Nagano and Nikaido, 2009) and penicillins (Lim and Nikaido, 2010). Efflux could also be stimulated by different kinds of substrates, including solvents (Kinana et al., 2013). The positive cooperativity in substrate efflux could be explained by the simultaneous binding of substrates to PBPs in the B or A monomers. Crystal structures of the AcrB-substrate complex revealed simultaneous substrate binding at DBP of B monomer and at PBP of A monomer (Nakashima et al., 2011; Eicher et al., 2012), implying that the substrate in PBP of A monomer more likely plays a role in stimulating the efflux. Simulations in this study provide the direct evidences that substrate binding at PBP of A monomer indeed stimulates substrate dissociation in $\mathrm{B}$ monomer through accelerating the conformational transitions according to the functional rotating mechanism. We noticed that the conformational changes were overall accelerated by the substrate in A monomer, not just the local changes in DBP (Figure 3). This is in line with the observation that the substrate would not dissociate from the DBP until most of the conformational changes completed during the functional rotation. Therefore, the substrate dissociation is allosterically regulated by the substrate binding in the neighboring monomer. Another possibility we could not rule out, however, is that ligand binding at $\mathrm{PBP}$ of the same monomer, i.e., in the B monomer, may also stimulate the substrate extrusion, although the corresponding intermediate state has not been detected in crystallography studies. Yet another mechanism of the positive cooperativity in substrate extrusion proposed by A. Kinana et al. is the direct interference of the stimulator with the substrate through competitive binding at the same DBP of B monomer (Kinana et al., 2013). It is worth noting that in the same study, decreases of Hill coefficients were observed in the presence of the stimulators (Kinana et al., 2013), which supports the allosteric mechanism with stimulators bound at different sites.

Another implication for the observation that substrate binding at A monomer promotes the protein conformational changes is that the functional rotation of AcrB trimer is partly energized by ligand binding in addition to the proton motive force. The accelerated conformational transition indicates that the free energy barrier of this process is lowered upon ligand binding. In other words, substrates are not only passively pumped by the molecular machine of AcrB, but also actively regulate the functional rotation of AcrB trimer. Recent combined crystallography and MD simulation study also suggested that drug binding is the driving force in the A to B (or L to T) transition (Mishra et al., 2014).

An unexpected observation in this work is that the functional rotation did not squeeze the PBP-bound substrate toward DBP during the A-B transition, rather it moved away toward the entrance. This might be attributed to the slight opening of the PC1-PC2 cleft upon A-B transition (Figure 5), suggesting that substrate translocation from $\mathrm{PBP}$ to $\mathrm{DBP}$ may require temporary closure of the cleft. In line with this, partially closed conformations of the cleft have been observed in monomer A by unbiased substrate-free MD simulations (Fischer and Kandt, 2013). An intermediate state with PC1-PC2 
cleft closed was also identified in another RND transporter ZneA (Pak et al., 2013). It is most likely that during the functional rotation, a similar intermediate state between A and B yet identified also exists in AcrB to prevent the back diffusion of the substrate.

Although our simulation was conducted on isolated AcrB, the RND transporter functions together with the periplasmic adaptor protein AcrA and outer membrane protein TolC as a tripartite assembly, and AcrA- $\mathrm{Mg}^{2+}$ has been reported to strongly accelerate the extrusion of substrate (Zgurskaya and Nikaido, 1999b). These findings raise an issue of whether the acceleration effect of multi-substrate binding observed in our simulation would still work after the association of AcrA- $\mathrm{Mg}^{2+}$. The crystal structure of heavy-metal efflux complex CusBA (Su et al., 2011), a homology of $A c r A B$, and the recently reported pseudo-atomic structure of AcrAB-TolC complex (Du et al., 2014) provide a valuable insight into the acceleration effect of AcrA- $\mathrm{Mg}^{2+}$. The structures show that the adaptor protein (CusB or AcrA) forms interactions with the TolC-docking domain and the subdomains PN2, PC1, and PC2 of the RND transporter. A noteworthy feature of the interactions between adaptor protein and RND transporter is that the subdomain PC2 forms much weaker contacts with the adaptor protein than PN2 and PC1 do. Only two residues on PC2 (Arg705 and Leu714) are located within $4 \AA$ of the adaptor protein and they form weak van der Waals interactions. The binding of adaptor protein may restrain the motions in $\mathrm{PN} 2$ and $\mathrm{PC} 1$, but is less likely to disturb the motions of $\mathrm{PC} 2$ and the relative motions

\section{References}

Cheng, X., Wang, H., Grant, B., Sine, S. M., and McCammon, J. A. (2006). Targeted molecular dynamics study of c-loop closure and channel gating in nicotinic receptors. PLoS Comput. Biol. 2:e134. doi: 10.1371/journal.pcbi.00 20134

Collu, F., Vargiu, A. V., Dreier, J., Cascella, M., and Ruggerone, P. (2012). Recognition of imipenem and meropenem by the rnd-transporter mexb studied by computer simulations. J. Am. Chem. Soc. 134, 19146-19158. doi: $10.1021 /$ ja307803m

Compoint, M., Picaud, F., Ramseyer, C., and Girardet, C. (2005). Targeted molecular dynamics of an open-state kcsa channel. J. Chem. Phys. 122, 134707. doi: $10.1063 / 1.1869413$

Darden, T., York, D., and Pedersen, L. (1993). Particle mesh ewald - an n.Log(n) method for ewald sums in large systems. J. Chem. Phys. 98, 10089-10092. doi: $10.1063 / 1.464397$

Du, D., Wang, Z., James, N. R., Voss, J. E., Klimont, E., Ohene-Agyei, T., et al. (2014). Structure of the acrab-tolc multidrug efflux pump. Nature 509, 512-515. doi: $10.1038 /$ nature 13205

Eicher, T., Cha, H. J., Seeger, M. A., Brandstatter, L., El-Delik, J., Bohnert, J. A., et al. (2012). Transport of drugs by the multidrug transporter acrb involves an access and a deep binding pocket that are separated by a switch-loop. Proc. Natl. Acad. Sci. U.S.A. 109, 5687-5692. doi: 10.1073/pnas.1114944109

Elkins, C. A., and Nikaido, H. (2002). Substrate specificity of the rnd-type multidrug efflux pumps acrb and acrd of Escherichia coli is determined predominantly by two large periplasmic loops. J. Bacteriol. 184, 6490-6498. doi: 10.1128/JB.184.23.6490-6499.2002

Feller, S. E., Zhang, Y. H., Pastor, R. W., and Brooks, B. R. (1995). Constantpressure molecular-dynamics simulation - the langevin piston method. J. Chem. Phys. 103, 4613-4621. doi: 10.1063/1.470648

Feller, S. E., Yin, D. X., Pastor, R. W., and MacKerell, A. D. (1997). Molecular dynamics simulation of unsaturated lipid bilayers at low hydration: between PC1 and PC2. Since the second substrate in monomer A binds between the PC1 and PC2 subdomains, the adaptor protein may have little influence on the behavior of the second substrate and the accelerated conformational changes brought by its binding. The acceleration effects of AcrA- $\mathrm{Mg}^{2+}$ and multi-substrate binding may operate simultaneously when AcrB functions. It is worth noting, however, that the above notions are largely derived based on the static structures of CusBA and AcrAB-TolC complexes. Further studies, especially those concerning dynamic properties, are still required to provide a fuller understanding on the molecular mechanism of the acceleration effects.

\section{Acknowledgments}

This work was supported by National Major Basic Research Program of China (2011CB808505, 2014CB910201), National Science Foundation of China $(21473034,21403036)$, Specialized Research Fund for the Doctoral Program of Higher Education (20130071140004), Science \& Technology Commission of Shanghai Municipality (08DZ2270500). We thank the super computer center of Fudan University for their allocation of computer time.

\section{Supplementary Material}

The Supplementary Material for this article can be found online at: http://www.frontiersin.org/journal/10.3389/fmicb. 2015.00302/abstract

parameterization and comparison with diffraction studies. Biophys. J. 73, 2269-2279. doi: 10.1016/S0006-3495(97)78259-6

Feng, Z., Hou, T., and Li, Y. (2012). Unidirectional peristaltic movement in multisite drug binding pockets of acrb from molecular dynamics simulations. Mol. Biosyst. 8, 2699-2709. doi: 10.1039/c2mb25184a

Fischer, N., and Kandt, C. (2011). Three ways in, one way out: Water dynamics in the trans-membrane domains of the inner membrane translocase acrb. Proteins 79, 2871-2885. doi: 10.1002/prot.23122

Fischer, N., and Kandt, C. (2013). Porter domain opening and closing motions in the multi-drug efflux transporter acrb. Biochim. Biophys. Acta 1828, 632-641. doi: 10.1016/j.bbamem.2012.10.016

Higgins, C. F. (2007). Multiple molecular mechanisms for multidrug resistance transporters. Nature 446, 749-757. doi: 10.1038/nature05630

Humphrey, W., Dalke, A., and Schulten, K. (1996). Vmd: visual molecular dynamics. J. Mol. Graph. 14, 33-38. doi: 10.1016/0263-7855(96) 00018-5

Husain, F., and Nikaido, H. (2010). Substrate path in the acrb multidrug efflux pump of Escherichia coli. Mol. Microbiol. 78, 320-330. doi: 10.1111/j.13652958.2010.07330.x

Jorgensen, W. L., Chandrasekhar, J., Madura, J. D., Impey, R. W., and Klein, M. L. (1983). Comparison of simple potential functions for simulating liquid water. J. Chem. Phys. 79, 926-935. doi: 10.1063/1.445869

Kandt, C., Ash, W. L., and Tieleman, D. P. (2007). Setting up and running molecular dynamics simulations of membrane proteins. Methods 41, 475-488. doi: 10.1016/j.ymeth.2006.08.006

Kinana, A. D., Vargiu, A. V., and Nikaido, H. (2013). Some ligands enhance the efflux of other ligands by the Escherichia coli multidrug pump acrb. Biochemistry 52, 8342-8351. doi: 10.1021/bi401303v

Kong, Y., Shen, Y., Warth, T. E., and Ma, J. (2002). Conformational pathways in the gating of Escherichia coli mechanosensitive channel. Proc. Natl. Acad. Sci. U.S.A. 99, 5999-6004. doi: 10.1073/pnas.0920 51099 
Koronakis, V., Sharff, A., Koronakis, E., Luisi, B., and Hughes, C. (2000). Crystal structure of the bacterial membrane protein tolc central to multidrug efflux and protein export. Nature 405, 914-919. doi: 10.1038/35016007

Lim, S. P., and Nikaido, H. (2010). Kinetic parameters of efflux of penicillins by the multidrug efflux transporter acrab-tolc of Escherichia coli. Antimicrob. Agents Chemother. 54, 1800-1806. doi: 10.1128/AAC.01714-09

Ma, J., and Karplus, M. (1997). Molecular switch in signal transduction: reaction paths of the conformational changes in ras p21. Proc. Natl. Acad. Sci. U.S.A. 94, 11905-11910. doi: 10.1073/pnas.94.22.11905

MacKerell, A. D., Bashford, D., Bellott, M., Dunbrack, R. L., Evanseck, J. D., Field, M. J., et al. (1998). All-atom empirical potential for molecular modeling and dynamics studies of proteins. J. Phys. Chem. B 102, 3586-3616. doi: $10.1021 /$ jp973084f

MacKerell, A. D., Feig, M., and Brooks, C. L. (2004). Extending the treatment of backbone energetics in protein force fields: limitations of gas-phase quantum mechanics in reproducing protein conformational distributions in molecular dynamics simulations. J. Comput. Chem. 25, 1400-1415. doi: 10.1002/jcc.20065

Martyna, G. J., Tobias, D. J., and Klein, M. L. (1994). Constant-pressure moleculardynamics algorithms. J. Chem. Phys. 101, 4177-4189. doi: 10.1063/1.467468

Mikolosko, J., Bobyk, K., Zgurskaya, H. I., and Ghosh, P. (2006). Conformational flexibility in the multidrug efflux system protein acra. Structure 14, 577-587. doi: 10.1016/j.str.2005.11.015

Mishra, S., Verhalen, B., Stein, R. A., Wen, P. C., Tajkhorshid, E., and McHaourab, H. S. (2014). Conformational dynamics of the nucleotide binding domains and the power stroke of a heterodimeric abc transporter. Elife 3:e02740. doi: 10.7554/eLife.02740

Miyamoto, S., and Kollman, P. A. (1992). Settle - an analytical version of the shake and rattle algorithm for rigid water models. J. Comput. Chem. 13, 952-962. doi: $10.1002 /$ jcc. 540130805

Morris, G. M., Huey, R., Lindstrom, W., Sanner, M. F., Belew, R. K., Goodsell, D. S., et al. (2009). Autodock4 and autodocktools4: automated docking with selective receptor flexibility. J. Comput. Chem. 30, 2785-2791. doi: 10.1002/jcc.21256

Murakami, S., Nakashima, R., Yamashita, E., and Yamaguchi, A. (2002). Crystal structure of bacterial multidrug efflux transporter acrb. Nature 419, 587-593. doi: $10.1038 /$ nature 01050

Murakami, S., Nakashima, R., Yamashita, E., Matsumoto, T., and Yamaguchi, A. (2006). Crystal structures of a multidrug transporter reveal a functionally rotating mechanism. Nature 443, 173-179. doi: 10.1038/nature05076

Nagano, K., and Nikaido, H. (2009). Kinetic behavior of the major multidrug efflux pump acrb of Escherichia coli. Proc. Natl. Acad. Sci. U.S.A. 106, 5854-5858. doi: 10.1073/pnas. 0901695106

Nakashima, R., Sakurai, K., Yamasaki, S., Nishino, K., and Yamaguchi, A. (2011). Structures of the multidrug exporter acrb reveal a proximal multisite drugbinding pocket. Nature 480, 565-569. doi: 10.1038/nature10641

Nikaido, H. (1996). Multidrug efflux pumps of gram-negative bacteria. J. Bacteriol. $178,5853-5859$.

Pak, J. E., Ekende, E. N., Kifle, E. G., O'Connell, J. D. 3rd, De Angelis, F., Tessema, M. B., et al. (2013). Structures of intermediate transport states of znea, a zn(ii)/proton antiporter. Proc. Natl. Acad. Sci. U.S.A. 110, 18484-18489. doi: 10.1073/pnas. 1318705110

Phillips, J. C., Braun, R., Wang, W., Gumbart, J., Tajkhorshid, E., Villa, E., et al. (2005). Scalable molecular dynamics with namd. J. Comput. Chem. 26, 1781-1802. doi: $10.1002 /$ jcc.20289

Pos, K. M. (2009). Drug transport mechanism of the acrb efflux pump. Biochim. Biophys. Acta 1794, 782-793. doi: 10.1016/j.bbapap.2008.12.015

Rappolt, M., Hickel, A., Bringezu, F., and Lohner, K. (2003). Mechanism of the lamellar/inverse hexagonal phase transition examined by high resolution $\mathrm{x}$-ray diffraction. Biophys. J. 84, 3111-3122. doi: 10.1016/S0006-3495(03)70036-8

Ruggerone, P., Vargiu, A. V., Collu, F., Fischer, N., and Kandt, C. (2013). Molecular dynamics computer simulations of multidrug rnd efflux pumps. Comput. Struct. Biotechnol. J. 5:e201302008. doi: 10.5936/csbj.201302008

Schlitter, J., Engels, M., Kruger, P., Jacoby, E., and Wollmer, A. (1993). Targeted molecular-dynamics simulation of conformational change - application to the t $-\mathrm{r}$ transition in insulin. Mol. Simulat. 10, 291. doi: 10.1080/089270293080 22170

Schulz, R., Vargiu, A. V., Collu, F., Kleinekathofer, U., and Ruggerone, P. (2010). Functional rotation of the transporter acrb: insights into drug extrusion from simulations. PLoS Comput. Biol. 6:e1000806. doi: 10.1371/journal.pcbi.1000806
Schulz, R., Vargiu, A. V., Ruggerone, P., and Kleinekathofer, U. (2011). Role of water during the extrusion of substrates by the efflux transporter acrb. J. Phys. Chem. B 115, 8278-8287. doi: 10.1021/jp200996x

Seeger, M. A., Schiefner, A., Eicher, T., Verrey, F., Diederichs, K., and Pos, K. M. (2006). Structural asymmetry of acrb trimer suggests a peristaltic pump mechanism. Science 313, 1295-1298. doi: 10.1126/science.1131542

Seeger, M. A., von Ballmoos, C., Eicher, T., Brandstatter, L., Verrey, F., Diederichs, K., et al. (2008). Engineered disulfide bonds support the functional rotation mechanism of multidrug efflux pump acrb. Nat. Struct Mol. Biol. 15, 199-205. doi: $10.1038 / \mathrm{nsmb} .1379$

Seeger, M. A., von Ballmoos, C., Verrey, F., and Pos, K. M. (2009). Crucial role of asp408 in the proton translocation pathway of multidrug transporter acrb: evidence from site-directed mutagenesis and carbodiimide labeling. Biochemistry 48, 5801-5812. doi: 10.1021/bi900446j

Sennhauser, G., Amstutz, P., Briand, C., Storchenegger, O., and Grutter, M. G. (2007). Drug export pathway of multidrug exporter acrb revealed by darpin inhibitors. PLoS Biol. 5:e7. doi: 10.1371/journal.pbio.0050007

Smart, O. S., Neduvelil, J. G., Wang, X., Wallace, B. A., and Sansom, M. S. (1996). Hole: a program for the analysis of the pore dimensions of ion channel structural models. J. Mol. Graph. 14, 354-360. doi: 10.1016/S0263-7855(97)00009-X

Su, C. C., Li, M., Gu, R., Takatsuka, Y., McDermott, G., Nikaido, H., et al. (2006). Conformation of the acrb multidrug efflux pump in mutants of the putative proton relay pathway. J. Bacteriol. 188, 7290-7296. doi: 10.1128/JB.00684-06

Su, C. C., Long, F., Zimmermann, M. T., Rajashankar, K. R., Jernigan, R. L., and Yu, E. W. (2011). Crystal structure of the cusba heavy-metal efflux complex of Escherichia coli. Nature 470, 558-562. doi: 10.1038/nature09743

Takatsuka, Y., and Nikaido, H. (2006). Threonine-978 in the transmembrane segment of the multidrug efflux pump acrb of Escherichia coli is crucial for drug transport as a probable component of the proton relay network. J. Bacteriol. 188, 7284-7289. doi: 10.1128/JB.00683-06

Takatsuka, Y., and Nikaido, H. (2007). Site-directed disulfide cross-linking shows that cleft flexibility in the periplasmic domain is needed for the multidrug efflux pump acrb of Escherichia coli. J. Bacteriol. 189, 8677-8684. doi: 10.1128/JB.01127-07

Takatsuka, Y., and Nikaido, H. (2009). Covalently linked trimer of the acrb multidrug efflux pump provides support for the functional rotating mechanism. J. Bacteriol. 191, 1729-1737. doi: 10.1128/JB.01441-08

Tikhonova, E. B., and Zgurskaya, H. I. (2004). Acra, acrb, and tolc of Escherichia coli form a stable intermembrane multidrug efflux complex. J. Biol. Chem. 279, 32116-32124. doi: 10.1074/jbc.M402230200

van der Vaart, A., Ma, J., and Karplus, M. (2004). The unfolding action of groel on a protein substrate. Biophys. J. 87, 562-573. doi: 10.1529/biophys..103.037333

Vanommeslaeghe, K., Hatcher, E., Acharya, C., Kundu, S., Zhong, S., Shim, J., et al. (2010). Charmm general force field: a force field for drug-like molecules compatible with the charmm all-atom additive biological force fields. J. Comput. Chem. 31, 671-690. doi: 10.1002/jcc.21367

Vanommeslaeghe, K., and MacKerell, A. D. Jr. (2012). Automation of the charmm general force field (cgenff) i: Bond perception and atom typing. J. Chem. Inf. Model. 52, 3144-3154. doi: 10.1021/ci300363c

Vanommeslaeghe, K., Raman, E. P., and MacKerell, A. D. Jr. (2012). Automation of the charmm general force field (cgenff) ii: Assignment of bonded parameters and partial atomic charges. J. Chem. Inf. Model. 52, 3155-3168. doi: $10.1021 / \mathrm{ci} 3003649$

Vargiu, A. V., Collu, F., Schulz, R., Pos, K. M., Zacharias, M., Kleinekathofer, U., et al. (2011). Effect of the f610a mutation on substrate extrusion in the acrb transporter: explanation and rationale by molecular dynamics simulations. J. Am. Chem. Soc. 133, 10704-10707. doi: 10.1021/ja202666x

Vargiu, A. V., and Nikaido, H. (2012). Multidrug binding properties of the acrb efflux pump characterized by molecular dynamics simulations. Proc. Natl. Acad. Sci. U.S.A. 109, 20637-20642. doi: 10.1073/pnas.1218348109

Weng, J., Fan, K., and Wang, W. (2012). The conformational transition pathways of atp-binding cassette transporter btucd revealed by targeted molecular dynamics simulation. PLoS ONE 7:e30465. doi: 10.1371/journal.pone.00 30465

Weng, J. W., Fan, K. N., and Wang, W. N. (2010). The conformational transition pathway of atp binding cassette transporter msba revealed by atomistic simulations. J. Biol. Chem. 285, 3053-3063. doi: 10.1074/jbc.M109. 056432 
Yamane, T., Murakami, S., and Ikeguchi, M. (2013). Functional rotation induced by alternating protonation states in the multidrug transporter acrb: allatom molecular dynamics simulations. Biochemistry 52, 7648-7658. doi: $10.1021 /$ bi400119v

Yao, X. Q., Kimura, N., Murakami, S., and Takada, S. (2013). Drug uptake pathways of multidrug transporter acrb studied by molecular simulations and sitedirected mutagenesis experiments. J. Am. Chem. Soc. 135, 7474-7485. doi: $10.1021 /$ ja310548h

Zgurskaya, H. I., and Nikaido, H. (1999a). Acra is a highly asymmetric protein capable of spanning the periplasm. J. Mol. Biol. 285, 409-420. doi: 10.1006/jmbi.1998.2313

Zgurskaya, H. I., and Nikaido, H. (1999b). Bypassing the periplasm: reconstitution of the acrab multidrug efflux pump of Escherichia coli.
Proc. Natl. Acad. Sci. U.S.A. 96, 7190-7195. doi: 10.1073/pnas.96.13. 7190

Conflict of Interest Statement: The authors declare that the research was conducted in the absence of any commercial or financial relationships that could be construed as a potential conflict of interest.

Copyright (C) 2015 Wang, Weng and Wang. This is an open-access article distributed under the terms of the Creative Commons Attribution License (CC BY). The use, distribution or reproduction in other forums is permitted, provided the original author(s) or licensor are credited and that the original publication in this journal is cited, in accordance with accepted academic practice. No use, distribution or reproduction is permitted which does not comply with these terms. 